\title{
Atenção nutricional a indivíduos com doença inflamatória intestinal: o relato de uma experiência de educação e produção de conhecimento por meio da assistência à saúde. Nutritional care for individuals with inflammatory bowel disease: report of an experience of education and knowledge development through health care
}

Maria Eduarda Ribeiro José ${ }^{1}$ Bruna Cadete Martins ${ }^{2}$ Livia Cristina Silva Rodrigues ${ }^{3}$ Isabelle Cristine Lôpo dos Santos4 Thaís da Silva Ferreira5 Fabricia Junqueira das Neves ${ }^{6}$

1 Nutricionista. Universidade Federal do Estado do Rio de Janeiro (UNIRIO), Brasil. E-mail: mariaeduardarj11@hotmail.com 2 Discente. Universidade Federal do Estado do Rio de Janeiro (UNIRIO), Brasil. E-mail: brunacadetemartins@gmail.com 3 Nutricionista. Universidade Federal do Estado do Rio de Janeiro (UNIRIO), Brasil. E-mail: lilirodrigues_@hotmail.com 4 Discente. Universidade Federal do Estado do Rio de Janeiro (UNIRIO), Brasil. E-mail: lopo.isabelle@gmail.com 5 Docente. Universidade Federal do Estado do Rio de Janeiro (UNIRIO), Brasil. E-mail: thais.ferreira@unirio.br 6 Docente. Universidade Federal do Estado do Rio de Janeiro (UNIRIO), Brasil. E-mail: fabricia.junqueira@unirio.br

Recebido em: 03/10/2020 | Aprovado em: 21/10/2020 DOI: $10.12957 /$ interag.2020.51080

\section{Resumo}

A doença inflamatória intestinal (DII) tem um grande impacto na qualidade de vida e a alimentação apresenta papel fundamental no desenvolvimento e tratamento da mesma. $\mathrm{O}$ atendimento nutricional pode então contribuir de forma significativa para qualidade de vida de indivíduos com DII, desde que contemple toda a complexidade que o indivíduo traz, incluindo a realidade em que está inserido. Foram realizadas consultas individuais nas quais foram abordados vários aspectos, por meio de um protocolo específico. A troca de saberes com os indivíduos possibilitou aos profissionais e alunos do ambulatório um melhor entendimento das peculiaridades da DII. A valorização da escuta do indivíduo aumentou a adesão ao tratamento, já que o mesmo participou da construção do conhecimento e foi sensibilizado para a importância das mudanças.

Palavras-chave: Atendimento Nutricional; Educação em Saúde; Doença de Crohn; Colite Ulcerativa

\begin{abstract}
Inflammatory bowel disease (IBD) has a major impact on quality of life and nutrition has an important influence in the development and treatment of these diseases. Nutritional care may contribute significantly to the treatment of individuals with IBD, as long as they contemplate all the complexity that the patient brings, including the reality in which they are inserted. Individual appointment was carried out in whom several aspects were approached, through a specific protocol previously elaborated. The exchange of information with the patients enabled clinicians and students to better understand the peculiarities of IBD. The evaluation of patient listening increased adherence to treatment, since the patient participated in the construction of knowledge and was sensitized to the importance of the changes.
\end{abstract}

Keywords: Nutritional Care; Health Education; Crohn's Disease; Ulcerative Colitis

Área temática: Saúde.

Linha de extensão: Saúde humana; Segurança Alimentar e Nutricional. 


\section{Introdução}

Doença inflamatória intestinal (DII) é uma doença crônica que acomete o trato gastrointestinal e se caracteriza por inflamação crônica do intestino, de etiologia ainda não definitivamente esclarecida, mas com possível interação entre fatores genéticos, imunes e ambientais.

As formas mais comuns da DII são a doença de Crohn e a colite ulcerativa. Ambas ocorrem no mundo todo e representam sério problema de saúde, pois atingem preferencialmente pessoas jovens, cursam com recidivas frequentes e podem assumir formas clínicas de alta gravidade ${ }^{1}$. A evolução da DII acarreta desde pequeno desconforto gastrointestinal até importantes complicações para os indivíduos acometidos, afetando a qualidade de vida tanto no âmbito social e psicológico quanto profissional, além do impacto negativo na expectativa de vida destes indivíduos ${ }^{2}$.

O curso clínico da DII varia com o tempo de evolução e o local de acometimento, entre outros fatores. Essas enfermidades apresentam períodos de exacerbação e remissão de intensidade variável. As principais manifestações clínicas observadas são decorrentes do acometimento do trato gastrointestinal pela doença, como diarreia, constipação intestinal, dor abdominal, cólicas abdominais, flatulência, má absorção intestinal, náuseas, pirose e vômitos ${ }^{3}$.

A atividade da doença, caracterizada por processo inflamatório das lesões intestinais, além da anorexia, má absorção de nutrientes, estresse oxidativo, necessidades nutricionais aumentadas e medicamentos utilizados no tratamento podem levar o indivíduo à desnutrição. Entre 70 e $80 \%$ dos mesmos apresentam perda de peso no decorrer da doença, levando a algum grau de comprometimento nutricional ${ }^{3}$.

Entretanto, o excesso de peso corporal foi recentemente identificado como sendo o mais frequente transtorno nutricional que acomete indivíduos com DII. Limitações para a realização de exercício físico por esses indivíduos, já que os mesmos podem permanecer mais tempo acamados nos períodos mais críticos da doença, e a alimentação 
inadequada são apontados como fatores que diminuem o gasto energético e poderiam contribuir para o ganho de peso excessivo ${ }^{4}$.

Sendo assim, o cuidado nutricional é claramente importante no tratamento de indivíduos com DII e inclui prevenção/tratamento da desnutrição ou obesidade, da deficiência de micronutrientes, prevenção da osteoporose e promoção de crescimento e desenvolvimento infantis adequados ${ }^{4}$. Desta forma, a assistência nutricional de indivíduos com DII, que apresentam necessidades especiais, contribui para a promoção à saúde e qualidade de vida dos mesmos. Com isso, o cuidado nutricional a esses indivíduos atende ao propósito da atenção primária à saúde de responder à maior parte das necessidades de uma população, integrando ações preventivas e curativas mediante plena participação dos indivíduos e da comunidade ${ }^{5}$.

Relatos sobre atividades de extensão evidenciam benefícios para a população assistida, como fortalecimento do vínculo com os profissionais, melhoria da autoestima, da segurança, do conforto e do enfrentamento do estresse. $O$ mesmo também pode ser observado para os profissionais, como o transparecer de reflexões, desenvolvimento da escuta ativa, do acolhimento e enriquecimento da formação profissional. Os diversos benefícios podem ser observados em diferentes estratégias com resultados positivos, como oficinas terapêuticas, educação nutricional, orientações, práticas reflexivas, entre outras ${ }^{6,7}$.

Diante do exposto, o objetivo do presente estudo é descrever a atenção nutricional ambulatorial a indivíduos com DII de um hospital universitário do Rio de Janeiro, na forma de um relato de experiência de educação, aprendizado e produção de conhecimento por meio da assistência à saúde.

\section{Atenção nutricional a indivíduos com DII por meio da extensão universitária}

Em um projeto de extensão universitária, desenvolvido por docentes e discentes da Universidade Federal do Estado do Rio de Janeiro (UNIRIO) desde outubro de 2016, 
é realizado atendimento nutricional na forma de consultas individuais para os pacientes com DII do ambulatório de Gastroenterologia do Hospital Universitário Gaffrée e Guinle (HUGG), da UNIRIO. O projeto foi aprovado pelo Comitê de Ética da UNIRIO (CAAE: 60841716.2.0000.5285). No referido ambulatório, os indivíduos recebiam previamente atendimento da equipe médica e cuidados de enfermagem, sendo introduzida a atenção nutricional por meio do encaminhamento médico, como forma de ampliar o cuidado ao indivíduo e trabalhar de maneira interdisciplinar na formação dos alunos da UNIRIO. Por se tratar de uma doença na qual a alimentação tem papel fundamental e interfere no prognóstico dos indivíduos acometidos, é recomendado que, independentemente da fase da doença, eles sejam assistidos por profissional nutricionista ${ }^{4}$.

As vagas para participação no projeto foram divulgadas e distribuídas entre os alunos dos últimos anos do curso de graduação em Nutrição da Escola de Nutrição da UNIRIO, selecionados de acordo com seu desempenho no curso, cumprimento de disciplinas na área de Nutrição Clínica e disponibilidade. Não havendo limite máximo para permanência no projeto, as horas não estavam inseridas em nenhuma disciplina e eram contabilizadas como atividades complementares. Na reforma curricular do curso de Nutrição da UNIRIO em andamento, as atividades do projeto integram o processo de previsão como atividade extensionista no projeto pedagógico do curso.

Antes do início do atendimento, respeitando os princípios éticos contidos na Declaração de Helsinki, os pacientes eram convidados a lerem o TCLE, e só era dada continuidade caso o paciente aceitasse e assinasse o termo. A consulta de nutrição era então iniciada com uma avaliação nutricional completa, incluindo aspectos socioeconômicos (nível de escolaridade, ocupação e renda); clínicos (escore de atividade de doença, medicação utilizada, complicações associadas); laboratoriais (hemograma, perfil lipídico e glicídico, nível de resistência à insulina e marcadores inflamatórios); antropométricos (peso corporal, estatura, índice de massa corporal e composição corporal) e dietéticos (história alimentar). Vale ressaltar que protocolos elaborados especificamente para o atendimento dessa população foram previamente desenvolvidos 
em outro projeto de extensão do mesmo grupo na UNIRIO nos anos de 2016 e 2017. Eles são utilizados durante o atendimento abordando os aspectos descritos acima.

A partir destas informações foi realizado o diagnóstico do estado nutricional que serviu de base para a estimativa das necessidades nutricionais. Após discussão da equipe de nutrição com a equipe médica e de enfermagem do ambulatório sobre o caso e após escuta atenta do relato dos indivíduos sobre sua tolerância aos diferentes alimentos (sintomas apresentados e sua relação com a alimentação) foi possível identificar associações entre a alimentação e a evolução da doença. Reunindo essas informações ao conhecimento previamente adquirido sobre os diferentes tipos de alimentos que podem influenciar o desenvolvimento da DII e seus sintomas, era definida a conduta nutricional. O plano alimentar prescrito era desenvolvido com base nas diretrizes da Sociedade Europeia de Nutrição Enteral e Parenteral para o tratamento nutricional da DII ${ }^{4}$ com a plena participação do indivíduo acometido pela doença, considerando além dos aspectos acima descritos, também seu estilo de vida, condições socioeconômicas, viabilidade de adesão ao tratamento nutricional proposto, atividades desenvolvidas ao longo do dia e sua percepção quanto ao possível impacto da atenção nutricional na sua qualidade de vida. Essa forma de atenção nutricional aos usuários com DII foi estabelecida à luz da diretriz para projetos de extensão da UNIRIO, de interdisciplinaridade e interprofissionalidade para as ações extensionistas, buscando superar a dicotomia das visões holística e especializada ${ }^{8}$.

Os resultados das avaliações realizadas eram apresentados aos indivíduos, assim como a conduta nutricional. Ambos eram registrados em prontuário hospitalar ao qual toda a equipe multidisciplinar tinha acesso: além dos nutricionistas, médicos (professores, funcionários do HUGG, residentes e pós-graduandos), enfermeiros e alunos de graduação da Nutrição e da Medicina (internos, bolsistas e voluntários de extensão e pesquisa). Vale ressaltar que orientações nutricionais específicas para o estado nutricional e quadro clínico de cada indivíduo foram utilizados para complementar o plano alimentar proposto, como forma de abordar outros aspectos do tratamento nutricional. 
O atendimento nutricional acontecia no mesmo dia do atendimento médico e da enfermagem, evitando assim onerar custos dos indivíduos e suas famílias com transporte, facilitar o acesso à atenção nutricional e contribuir para adesão ao tratamento nutricional. Situações especiais que demandavam acompanhamento nutricional de alguns indivíduos com maior frequência por determinado período constituíam exceções a essa prática, sendo o retorno programado de acordo com a necessidade identificada pela equipe e com a disponibilidade do mesmo.

A participação da família no atendimento não era obrigatória, mas recorrente. Sempre que o indivíduo estava acompanhado de algum familiar, o mesmo era estimulado a participar do atendimento, o que contribuía para a compreensão e adesão às orientações, intensificava a troca de saberes e propiciava maior disseminação dos conhecimentos.

A adesão à conduta nutricional e ao plano alimentar construídos por meio do projeto era avaliada pelo relato dos indivíduos em relação: às mudanças alimentares; melhora da sintomatologia (dor, desconforto abdominal, flatulência, presença de sangue e muco nas fezes, função intestinal); melhora da qualidade de vida; e retorno ao atendimento nutricional.

Ao final dos atendimentos do dia, a equipe de nutrição se reunia e cada colaborador relatava sua experiência com os indivíduos acompanhados para discussão das condutas propostas e compartilhamento dos saberes adquiridos em cada encontro. Nesse momento era realizada avaliação do impacto das ações propostas por meio do acompanhamento dos seguintes aspectos: evolução do estado nutricional dos indivíduos; resposta ao tratamento clínico; períodos de exacerbação do quadro clínico; aparecimento de sintomas; adesão às orientações nutricionais; impacto sobre a qualidade de vida; número de internações hospitalares relacionadas a complicações da DII; e realização de procedimentos cirúrgicos relacionados à DII.

\section{Perfil dos indivíduos com DII acompanhados}


No período de outubro de 2016 a março de 2020, foram realizadas 428 consultas nutricionais, sendo 200 de primeira vez e 228 retornos. Foram atendidos 47\% (n=94) indivíduos com doença de Crohn, 46\% ( $n=91)$ com colite ulcerativa e 7\% ( $n=14)$ com formas não classificáveis de DII, que apresentaram média de idade de 50,6 anos (de 18 anos a 90 anos), sendo 69\% ( $\mathrm{n}=138)$ do sexo feminino. Quanto ao diagnóstico do estado nutricional, foram observados $8 \%(n=16)$ de indivíduos desnutridos e $54 \%(n=107)$ com excesso de peso.

A baixa prevalência de desnutrição é justificada possivelmente pelo tratamento clínico ou cirúrgico dos indivíduos acompanhados no ambulatório. A assistência à saúde por meio do acompanhamento com profissionais especializados e do acesso à medicação mais adequada ao tratamento parecem ser os principais fatores que impedem a deterioração do estado nutricional e o desenvolvimento da desnutrição no grupo acompanhado. Por outro lado, o excesso de peso corporal é confirmado então como o desvio nutricional mais frequente nesse grupo, como descrito anteriormente na literatura ${ }^{4}$. Desta forma, assim como na população em geral $^{9}$, os indivíduos com DII acompanhados no HUGG podem apresentar risco aumentado para o desenvolvimento de doenças relacionadas à obesidade, como diabetes mellitus, doenças cardiovasculares e câncer de cólon ${ }^{10}$. Além da menor realização de exercício físico e menor gasto calórico por parte desses indivíduos ${ }^{4}$, como mencionado anteriormente, acredita-se que o sucesso no tratamento clínico com a remissão dos principais sintomas resulte em melhora do estado geral e numa alimentação inadequada. Esse perfil alimentar, semelhante àquele observado na maioria da população brasileira, caracterizado pelo consumo de refeições de alta densidade calórica, ricas em açúcares simples, gordura total, saturada e trans, pobre em fibras e micronutrientes pode constituir outro importante fator que contribui para o ganho excessivo de peso na amostra estudada.

Apesar da baixa frequência observada de desnutrição, quando presente este desvio tem importantes repercussões negativas no desenvolvimento da doença e na qualidade de vida do indivíduo. Essa condição está associada ao retardo da melhora clínica e da recuperação pós-operatória, bem como à mortalidade e a maiores índices de complicações 
cirúrgicas. Sua ocorrência se deve não só à atividade da doença, mas também à anorexia, má absorção de nutrientes, estresse oxidativo, necessidades nutricionais aumentadas e uso de medicamentos ${ }^{3}$.

Nesse contexto, afirma-se a importância das diretrizes para o tratamento nutricional de indivíduos com DII publicadas recentemente, que ressaltam que os mesmos devem receber um atendimento nutricional individual baseado na sua situação pessoal específica. A atenção nutricional deve se dar de preferência com suporte ativo de um nutricionista dedicado como parte de uma abordagem multidisciplinar, que também deve incluir um gastroenterologista, cirurgião, enfermeiro, psicólogo, entre outros profissionais ${ }^{4}$, o que tem sido em grande parte alcançado no atual projeto.

Desta forma, o atendimento nutricional pode contribuir de forma significativa para o tratamento dos indivíduos com DII, desde que contemple toda a complexidade que o mesmo traz, incluindo a realidade em que está inserido. Todo esse conjunto de ações possibilita um melhor prognóstico da doença, promovendo impacto e transformação social por meio da melhora da qualidade de vida dos indivíduos acometidos.

\section{Extensão universitária viabilizando assistência nutricional especializada no tratamento das DII}

As ações realizadas até o momento pelo projeto de extensão incluem a atenção nutricional especializada para indivíduos com DII, com uma abordagem ampla que inclui diferentes aspectos da vida dos mesmos. O desenvolvimento das diferentes etapas do atendimento nutricional, como avaliação do estado nutricional, determinação das necessidades nutricionais orientações e prescrição nutricional de maneira personalizada é fundamental para o sucesso da atenção nutricional. Isso porque a apresentação das DII pode ocorrer de maneira muito diferente entre os indivíduos, com cada um deles apresentando necessidades muito específicas, que têm sido em grande parte atendidas pela atuação dos colaboradores do presente projeto de extensão. A atuação dos docentes e 
discentes dos cursos de graduação em Nutrição da UNIRIO tem, portanto, apoiado a assistência à saúde dos indivíduos com DII, tanto na fase ativa da doença (quando os sintomas estão presentes), quanto nos períodos de remissão da doença (quando poucos ou nenhum sintoma é observado).

As ações desenvolvidas por meio deste projeto de extensão universitária possibilitam aos indivíduos com DII escolhas alimentares que não causem exacerbação dos sintomas clínicos característicos da doença, assim como a recuperação/manutenção do estado nutricional adequado. Desta forma, essas ações geram impacto positivo no tratamento e controle das DII para a retomada de atividades produtivas e intelectuais, como o trabalho e o estudo, para o retorno ao convívio social e consequentemente para melhora da qualidade de vida dos indivíduos e suas famílias, assim como para redução das internações hospitalares e da realização de procedimentos cirúrgicos e dos custos hospitalares.

Em adição, as ações de extensão universitária realizadas colocam a comunidade acadêmica (docentes e discentes) em contato direto com o público que demanda uma atenção nutricional especializada, promovendo uma inter-relação da UNIRIO, mais precisamente através da Escola de Nutrição, com a sociedade. Em resumo, todas essas ações promovem impacto e transformação social por meio da melhora da qualidade de vida dos indivíduos envolvidos, assim como de seus familiares e da sociedade em que vivem.

\section{Educação e aprendizado por meio da assistência à saúde}

A escuta atenta do relato dos indivíduos com DII sobre sua tolerância gastrointestinal aos diferentes tipos de alimentos e sua percepção sobre a intensidade dos sintomas relacionados à ingestão desses alimentos é capaz de contribuir de forma significativa para a construção do conhecimento por parte da equipe multiprofissional. Isso porque com base em diversas observações os colaboradores são capazes de identificar 
alimentos geralmente envolvidos no surgimento de sintomas ou na evolução do quadro clínico da doença, além daqueles tradicionalmente descritos na literatura científica. Para tanto, o estabelecimento da interação dialógica com o indivíduo é imprescindível. Esse tipo de diálogo, que permite a troca de saberes para a construção do conhecimento tem se configurado como uma valiosa experiência de educação e aprendizado para os docentes e discentes envolvidos no projeto. No desenvolvimento dessas ações de extensão, esse tipo de diálogo se desenvolve não só entre os indivíduos e os profissionais de saúde, mas também entre docentes e discentes, e entre os profissionais das diversas áreas da saúde envolvidos, desconstruindo o modelo hierarquizado de transmissão de conhecimento.

Em adição, ao retornar ao seu ambiente social, familiar e de trabalho, os indivíduos com DII assistidos pelo projeto tornam-se propagadores dos conceitos e ideias abordados durante o atendimento, e retornam nos encontros posteriores com novas demandas que estimulam ainda mais a equipe envolvida, promovendo o desenvolvimento de estratégias para atender às novas questões apresentadas. $\mathrm{O}$ resultado é o enriquecimento da experiência para todos os envolvidos, a partir das vivências de cada um.

Todo esse processo contribui para a sensibilização do indivíduo atendido. E, essa sensibilização é fundamental para que ocorra a adesão ao plano alimentar proposto, já que muitas vezes existe uma demanda por mudanças importantes no estilo de vida destes indivíduos. Como nesse caso, a conduta nutricional é construída juntamente com o indivíduo, o mesmo se apropria dessa intervenção aumentando sua adesão ao tratamento. Vale ressaltar que a sensibilização dos indivíduos para a importância das mudanças também permite a reafirmação e materialização dos compromissos éticos e solidários da universidade pública brasileira.

A atenção à saúde desenvolvida de maneira interprofissional e interdisciplinar faz com que o indivíduo seja avaliado e tratado como um todo, abordando a complexidade que esse tipo de atendimento envolve e que vai desde a história patológica pregressa, todos os sinais, sintomas e queixas do indivíduo, avaliação nutricional até chegar à prescrição nutricional, englobando as preferências, escolhas e aversões sinalizadas pelo 
próprio e colaborando para a construção coletiva da conduta. Desta forma, rompe-se então com a visão fragmentada de atendimento, proporcionando ao indivíduo um atendimento menos tenso, mais seguro, afetuoso e terno. Esse tipo de prática também promove para os discentes envolvidos no projeto, impacto na sua formação, mostrando que um cuidar autêntico ao indivíduo, e não um simples tratar permite a este exteriorizar suas necessidades na busca de soluções, com ênfase em sua individualidade ${ }^{11}$.

Essa percepção de valorização da escuta do indivíduo também contribui para a transformação da realidade destes e de suas famílias, assim como para a autonomia do indivíduo acometido por uma enfermidade de caráter crônico. A melhora da qualidade do atendimento do ambulatório, outra vantagem observada com a metodologia proposta neste projeto, também influencia positivamente a formação dos discentes.

Como dito anteriormente, o presente projeto de extensão envolve discentes do curso de graduação em Nutrição da UNIRIO, que prevê em sua matriz curricular a possibilidade de desenvolvimento de ações de extensão de maneira complementar e está caminhando para a incorporação de ações de extensão nas disciplinas do curso. No referido ambulatório atuam ainda internos e residentes médicos, que também são enriquecidos na sua formação pela atuação numa equipe interdisciplinar. Fica então evidente a vertente do ensino presente no atual projeto, que em associação com produção de dados de pesquisa científica caracterizam o caráter multidimensional das ações. Desta forma, os três pilares indissociáveis da universidade pública, ensino, pesquisa e extensão estão contemplados e são fundamentais para a realização do presente projeto.

\section{Produção de conhecimento por meio da assistência à saúde}

A experiência de 42 meses neste projeto de extensão permitiu à equipe multidisciplinar identificar a carência por parte dos indivíduos com DII e suas famílias de materiais educativos confiáveis e de fácil compreensão sobre a relação entre alimentação e nutrição e DII. Esta carência estimulou o desenvolvimento de outro projeto de extensão com objetivo de produzir materiais voltados para a população com DII e seus familiares. 
Este projeto, desenvolvido pela mesma equipe encontra-se em andamento e, a partir desta experiência de assistência à saúde, publicou o material educativo com o tema alimentação, nutrição e DII disponibilizado no site da Escola de Nutrição da UNIRIO (http://www.unirio.br/ccbs/nutricao/niden/arquivo/alimentacao-na-doenca-inflamatoriaintestinal). Vale lembrar que este material educativo não substitui o acompanhamento com nutricionista, sendo seu objetivo principal contribuir para o esclarecimento de possíveis dúvidas e dar autonomia para escolhas alimentares mais adequadas e saudáveis.

Considerando a escassez de dados referentes ao estado nutricional de indivíduos com DII no Brasil, o atendimento nutricional desenvolvido a partir deste projeto de extensão viabilizou o desenvolvimento de projeto de pesquisa que visa traçar o perfil nutricional dos indivíduos acompanhados. Esses dados poderão contribuir para a caracterização da população atendida, assim como para o preenchimento da lacuna sobre o impacto desses agravos no estado nutricional dos indivíduos brasileiros acometidos pela DII, possibilitando abordagem adequada e direcionada para os principais distúrbios nutricionais observados.

\section{Considerações Finais}

Conclui-se que a atenção nutricional aos indivíduos com DII é de primordial importância, já que a mesma permite um melhor prognóstico da doença, além de promover impacto e transformação social através da melhora da qualidade de vida dos indivíduos portadores de DII. Em adição, os docentes e discentes envolvidos no atual projeto também aprimoram características importantes para que haja uma troca de saberes com os indivíduos portadores de DII, assim como a desconstrução do modelo hierarquizado de transmissão de conhecimento, valorizando uma escuta ativa e o acolhimento. Vale ressaltar que a interação com a equipe multiprofissional e a desfragmentação do atendimento também permite melhores resultados para esses indivíduos. 
Para todos os envolvidos no atual projeto, fica o compromisso de se tornar um multiplicador do conhecimento não só sobre o tratamento nutricional da doença, mas também de uma vida saudável em seu ambiente profissional, familiar e social.

\section{Contribuições individuais}

MERJ: interpretação dos resultados, redação do artigo, aprovação da versão a ser publicada

BCM: interpretação dos resultados, redação do artigo, aprovação da versão a ser publicada

LCSR: interpretação dos resultados, redação do artigo, aprovação da versão a ser publicada

ICLS: interpretação dos resultados, revisão crítica do artigo, aprovação da versão a ser publicada

TSF: concepção e planejamento do estudo, revisão crítica do artigo, aprovação da versão a ser publicada

FJN: concepção e planejamento do estudo, revisão crítica do artigo, aprovação da versão a ser publicada

\section{Referências}

1. SOUZA M. M., BELASCO A. G. S., NASCIMENTO J. E. A. Perfil Epidemiológico dos Pacientes Portadores de Doença Inflamatória Intestinal do Estado de Mato Grosso. Revista brasileira Coloproctologia. Mato Grosso, Cuiabá, v.28, n.3, Julho/Setembro, 2008.

2. ZALTMAN C. Doença inflamatória intestinal: qual a relevância da doença no Brasil? Editorial. Caderno de Saúde Pública. Rio de Janeiro, v.23, n.5, Maio, 2007.

3. SANTOS L. A. A., DORNA M. S., VULCANO D. S. B., AUGUSTI L., FRANZONI L. C., GONDO F. F., ROMEIRO F. G., SASSAKI L. Y. Terapia nutricional nas doenças inflamatórias intestinais: artigo de revisão. Nutrire. Botucatu, SP, v.40, n.3, Dezembro, 2015. 
4. FORBES A., ESCHER J., H_EBUTERNE X., KłE S., KRZNARIC Z., SCHNEIDER S., SHAMIR R., STARDELOVA K., WIERDSMA N., WISKIN A. E., BISCHOFF S. C. ESPEN guideline: Clinical nutrition in inflammatory bowel disease. Clinical Nutrition v. 36, p. 321-347, 2017.

5. MENDES E. V. A construção social da atenção primária à saúde. Brasília: Conselho Nacional de Secretários de Saúde - CONASS, 2015.

6. FRANÇA F.C., SANCHES L.C., GARBELINI M.C.L. Ações de extensão universitária: educação nutricional para idosas. Interagir: pensando a extensão. N.28, p.58-79, 2019.

7. SOUZA M.M.L., SOUZA S.V., MELLO R. Oficinas terapêuticas na atenção primária: um relato de experiência. Raízes e Rumos. N.5, p.217-222, 2017.

8. FORPROEX. FÓRUM DE PRÓ-REITORES DE EXTENSÃO DAS UNIVERSIDADES PÚBLICAS BRASILEIRAS. Plano Nacional de Extensão Universitária. MEC e SESu: 2010.

9. IBGE - Instituto Brasileiro de Geografia e Estatística. Pesquisa de Orçamentos Familiares 2008-2009 - POF. Rio de Janeiro, 2010.

10.SUIBHNE T., RAFTERY T.C., MCMAHON O., WALSH C., O'MORAIN C., O'SULLIVAN M. High prevalence of overweight and obesity in adults with Crohn's disease: associations with disease and lifestyle factors. Journal Crohns Colitis. V.7, n.7, p. 241-248, August, 2013.

11. MORAIS G. S. N., COSTA S. F. G., FONTES W.D., CARNEIRO A.D. Comunicação como instrumento básico no cuidar humanizado em enfermagem ao paciente hospitalizado. Acta Paul Enferm. V.22, n.3, p.323-327, 2009. 